\title{
Bragg-mirror-like circular dichroism in bio-inspired quadruple-gyroid 4srs nanostructures
}

\author{
Benjamin P Cumming ${ }^{1,2}$, Gerd E Schröder-Turk ${ }^{3}$, Sukanta Debbarma ${ }^{4}$ and Min $\mathrm{Gu}^{1,2}$
}

The smooth and tailorable spectral response of Bragg mirrors has driven their pervasive use in optical systems requiring customizable spectral control of beam propagation. However, the simple nature of Bragg mirror reflection prevents their application to the control of important polarization states such as circular polarization. While helical and gyroid-based nanostructures exhibiting circular dichroism have been developed extensively to address this limitation, they are often restricted by the spectral inconsistency of their optical response. Here we present the fabrication and characterization of quadruple-gyroid 4srs nanostructures exhibiting bio-inspired Bragg-mirror-like circular dichroism: a smooth and uniform band of circular dichroism reminiscent of the spectrum of a simple multilayer Bragg-mirror. Furthermore, we demonstrate that the circular dichroism produced by 4srs nanostructures are robust to changes in incident angle and beam collimation, providing a new platform to create and engineer circular dichroism for functional circular polarization manipulation.

Light: Science \& Applications (2017) 6, e16192; doi:10.1038/lsa.2016.192; published online 13 January 2017

Keywords: Bio-inspired; Bragg-mirror; Chirality; Circular dichroism; Gyroid

\section{INTRODUCTION}

Bragg mirrors are essential building blocks of many optical systems due to their ability to achieve near-total reflection of light across a precisely defined band of wavelengths. Formed by the constructive interference of successive Fresnel reflections from within a simple multilayer dielectric stack ${ }^{1,2}$, the reflection spectra of Bragg mirrors at normal incidence are characterized by a smooth, uniform and strong band of unpolarized reflection surrounded by high transmission ${ }^{3}$. Through these tailorable reflection properties, Bragg mirrors have found use in numerous applications requiring precise spectral control of beam propagation such as dielectric mirrors, beamsplitters and filters ${ }^{4}$, fibre optic sensors and lasers ${ }^{5}$, and in many other distributed feedback laser systems ${ }^{6}$.

While the prevalence of devices facilitated by the reflection from Bragg mirrors is noteworthy, a growing demand to manipulate specific polarization states ${ }^{7,8}$ has driven a focus on nanostructures capable of normal incidence interaction with a particular polarization. Specifically, a demand for control of spin angular momentum carrying circular polarization ${ }^{8-10}$ has led to the development of chiral nanostructures such as helical spiral arrays ${ }^{11-19}$ and gyroids ${ }^{20-23}$ that exhibit circular dichroism: a differential reflection, transmission or absorption between left circularly polarized (LCP) and right circularly polarized (RCP) light. However, even though these structures can exhibit circular dichroism, their spectral behaviour is often dominated by sharp and narrow features ${ }^{13-21}$, unpolarized reflection bands ${ }^{16-18,22}$, and fluctuations in the sign of the circular dichroism ${ }^{17-22}$ when a Bragg-mirror-like response, defined here as a spectral response comparable to the simple, clean and smooth spectrum of a Bragg mirror, would be preferred.

In this article we demonstrate the presence of bio-inspired Braggmirror-like (Bragg-like for short) circular dichroism in quadruplegyroid 4srs nanostructures that transmit only a single handedness of circular polarization. Taking inspiration from the strong but unpolarized Bragg-like reflections produced by the aligned single-gyroid 1srs-based wing-scales of the butterfly Parides sesostris, we utilize the laser direct write technique to create and align new 4srs nanostructures that exhibit smooth and strong circularly polarized transmission spectra reminiscent of the spectral response of a simple multilayer Bragg-mirror, and with similarities in its physical origin. We reveal a strong robustness of the circular dichroism band to variations in incident angle and beam collimation, and demonstrate the ability to incorporate defect modes within the circularly polarized stop-band that may enable the development of new chiral photonic devices.

\section{MATERIALS AND METHODS}

Crystallographic notation

Standard crystallographic notation with Miller indices is used to describe individual and families of directions and planes. The notations $\langle x y z>$ and $[x y z]$ are used to denote a family of directions equivalent by symmetry, and individual directions, respectively.

\footnotetext{
${ }^{1}$ Laboratory of Artificial-Intelligence Nanophotonics and CUDOS, School of Science, RMIT University, Melbourne, Victoria 3001 , Australia; ${ }^{2}$ Centre for Micro-Photonics, Faculty of Science, Engineering and Technology, Swinburne University of Technology, Hawthorn, Victoria 3122, Australia; ${ }^{3}$ School of Engineering and Information Technology, Mathematics and Statistics, Murdoch University, Murdoch, Western Australia 6150, Australia and ${ }^{4}$ Laser Physics Centre and CUDOS, Research School of Physical Sciences and Engineering, Australian National University, Canberra, Australian Capital Territory 2601, Australia

Correspondence: M Gu, Email: min.gu@rmit.edu.au

Received 5 April 2016; revised 11 July 2016; accepted 18 July 2016; accepted article preview online 3 June 2016
} 
The notation $(x y z)$ is used to denote an individual plane that is orthogonal to the direction $[x y z]$.

\section{Numerical simulations}

Calculation of photonic band-structure modes is performed using the open source software package MPB, developed by MIT. The 4 srs and 1srs nanostructures are constructed within simple cubic (SC) and body centred cubic (BCC) primitive unit cells, respectively, from cylindrical or elliptical line segments terminated by spherical and elliptical caps. Each nano-structure is discretized across the lattice vectors with a grid resolution of 64 pixels per lattice unit, and the dielectric function is averaged over a mesh size of 8 at each grid point. After solution of each frequency eigenvalue $(\omega)$, the corresponding magnetic eigenfield $\left(H_{k, n}^{*}\right)$ with wave vector $k$ is analysed for its degree of circular dichroism and for its coupling amplitude to a wave of arbitrary polarization ${ }^{24,25}$. To begin, the coupling amplitude between incident circularly polarized plane waves with wave vector $q \| k$ and the magnetic eigenfield in planes $P$ orthogonal to $k$ are computed via the overlap integrals

$$
C_{k, n}^{ \pm}=\left|\frac{1}{\iint_{P} d P} \iint_{P} \frac{1}{\sqrt{2}}\left(\hat{\mathbf{e}}_{1} \pm i \hat{\mathbf{e}}_{2}\right) \cdot H_{k, n}^{*}(P)\right|^{2}
$$

where $\frac{1}{\sqrt{2}}\left(\hat{\mathbf{e}}_{1} \pm i \hat{\mathbf{e}}_{2}\right)$ are the normalized circularly polarized plane waves and $H_{k, n}^{\sqrt{2}}(P)$ is the normalized complex conjugate of the eigenfield in the plane $P$. The unit vectors $\hat{e}_{1}$ and $\hat{e}_{2}$ form an orthogonal set with $k$.

The degree of circular dichroism, $C$, which is termed the circular dichroism index, is given by the definition taken from the literature ${ }^{24-27}$

$$
C_{k, n}=\operatorname{sgn}\left(q \cdot \nabla_{k} \omega\right)\left(\frac{C_{k, n}^{+}-C_{k, n}^{-}}{C_{k, n}^{+}+C_{k, n}^{-}}\right)
$$

where sgn is the sign function. Similarly, the amplitude of coupling the mode to a wave of arbitrary polarization, $\beta$, which is termed the coupling index, is also given by the definition taken from the literature ${ }^{24-27}$

$$
\beta_{k, n}=C_{k, n}^{+}+C_{k, n}^{-}
$$

The circular dichroism and coupling indices are useful measures to quantify the nature of individual Bloch modes in terms of circular polarization, providing intuition for the full scattering problem that can only be solved when considering the complex band-structure including evanescent modes $^{28}$. We consider 64 different planes along $k$ within the magnetic eigenfield, averaging $C$ and $\beta$ across all the planes to reveal final mean values. The coupling index for each mode in the band diagrams is represented as the size of the plot while the circular dichroism index of each mode in the band-diagram is represented by the colour of the plot. Modes with $C<0.5$ are considered LCP and are plotted blue, while modes with $C>0.5$ are considered RCP and are plotted red. Modes with $\beta<0.1$ are considered low-coupling modes.

Numerical simulations of the transmission spectra are performed using the commercial finite element software CST Microwave Studio. The cubic non-primitive unit-cell is used to model the 1srs nanostructure transmission while the SC primitive unit-cell is used to model the 4 srs nanostructure. The $\alpha=5^{\circ}$ half apex angle illumination cone surrounding the [001] axis is modelled by a smoothed average of 95 plane wave simulations equally spaced over the cone of illumination.

\section{Thick film deposition}

Films of $\mathrm{As}_{2} \mathrm{~S}_{3}$ of $\approx 16 \mu \mathrm{m}$ thickness were prepared from stoichiometric $\mathrm{As}_{2} \mathrm{~S}_{3}$ bulk glass using thermal evaporation ${ }^{29}$. A temperature controlled Ta baffled boat housing the $\mathrm{As}_{2} \mathrm{~S}_{3}$ was held at a temperature of $310^{\circ} \mathrm{C}$ in a vacuum chamber pumped to a base pressure of $2 \times 10^{-7}$ torr. A carousel under planetary rotation at the top of the chamber held $170 \mu \mathrm{m}$ thick glass substrates at room temperature and captured the evaporating $\mathrm{As}_{2} \mathrm{~S}_{3}$ at a deposition rate of $\approx 0.6 \mathrm{~nm} \mathrm{~s}^{-1}$. In the as-deposited state, the $\mathrm{As}_{2} \mathrm{~S}_{3}$ films were measured to have an approximate refractive index of 2.35 that increases to 2.43 after annealing $^{30}$. Raman spectroscopy was used to confirm that the deposited films contained the weakly interconnected $\mathrm{As}_{4} \mathrm{~S}_{6}$ cage molecules that enable photo-polymerization ${ }^{29,31}$

\section{Laser nano-fabrication}

The full details of the adaptive optics enhanced direct laser writing system have been described elsewhere ${ }^{22}$. An amplified Ti:Sapphire femto-second laser, operating at a central wavelength of $800 \mathrm{~nm}$, a repetition rate of $10 \mathrm{kHz}$ and with a pulse duration of $100 \mathrm{fs}$ is expanded to fill the area of a liquid crystal-based SLM (HSPDM5120785-PCIe, Boulder Nonlinear Systems) that provided phase-only modulation of the laser beam for compensating systeminduced optical aberrations, as well as the strong spherical aberration that is imparted onto the beam when focusing through a glass coverslip and into the high refractive index $\mathrm{As}_{2} \mathrm{~S}_{3}$. At this wavelength, the deposited film is transparent and no photo-polymerization occurs. Tracing the focus along the path of the 4srs nets enables photopolymerization back to $\mathrm{As}_{2} \mathrm{~S}_{3}$ only of the 4 srs geometry and at any size and orientation that is desired. The use of adaptive optics in the fabrication system is essential to achieve high-quality nano-fabrication in $\mathrm{As}_{2} \mathrm{~S}_{3}$ films as strong spherical aberration that is imparted onto the focused laser beam by the mismatch in glass and $\mathrm{As}_{2} \mathrm{~S}_{3}$ refractive indices can significantly distort fabricated structures such as gyroids ${ }^{22}$. The reflected beam from the SLM was imaged to the back aperture of a high numerical aperture $(\mathrm{NA}=1.4)$ objective lens that was used to focus the beam into the $\mathrm{As}_{2} \mathrm{~S}_{3}$. A three-dimensional piezo-electric translation stage and mechanical shutter were used to translate the sample and switch the beam such that the focus of the laser traced out the network segments of the 4 srs within the $\mathrm{As}_{2} \mathrm{~S}_{3}$ film. The $4 \mathrm{srs}$ nanostructures were typically written at a speed of $10 \mu \mathrm{m} \mathrm{s}^{-1}$ and with an average laser power between 1.75 and $2.0 \mu \mathrm{W}$.

\section{Wet chemical etching}

After photo-polymerization, the $\mathrm{As}_{2} \mathrm{~S}_{3}$ thick films were immersed for $3 \mathrm{~min}$ in a $2 \mathrm{~mol}-\%$ ratio of diisopentylamine in dimethylsulfoxide at room temperature and with constant stirring. Immediately after etching, the films were immersed in acetone to cease the etching process.

\section{Transmission spectroscopy}

Transmission spectra were measured with a Fourier transform infrared spectrometer coupled to an infrared microscope. The microscope contained two Cassegrain reflection objectives for illumination and collection of transmitted light. Conversion of the broadband unpolarized light from the spectrometer to circular polarization was achieved via the addition of a broadband $(2.5-10 \mu \mathrm{m}) \mathrm{BaF}_{2}$ wire grid linear polarizer and a broadband $(2.5-7 \mu \mathrm{m}) \mathrm{MgF}_{2}$ circular polarizer. The incident beam was limited to a small cone of angles surrounding the [001] axis by placing a small aperture into the path of the objective lens, reducing the NA of the excitation from $0.68 \mathrm{NA}$ to $<0.1 \mathrm{NA}$ such that the beam extended over a cone with a half 
apex angle of $\alpha=5^{\circ}$. The measured spectra were corrected for measurement-induced spectral shifts and scattering losses, and smoothed to remove noise.

\section{RESULTS AND DISCUSSION}

\section{4srs intergrowth}

The 1 srs network (net) derives its name from the $\mathrm{SrSi}_{2}$ crystal ${ }^{32}$, within which the Si atoms form a single chiral network with cubic symmetry $I 4_{1} 32$. Inflating the 1 srs net into cylindrical segments of finite radius forms a 1srs nanostructure, also known as a single-gyroid, that is well suited to experimental fabrication ${ }^{20-22}$. A common unit-cell of the 1srs nanostructure is the truncated octahedron with BCC basis ${ }^{24,25,33,34}$, shown in Figure 1a. However, the only substantial circular dichroism within dielectric 1srs nanostructures ${ }^{24,25}$ is known to occur along the cubic $<100>$ axes (see Materials and Methods for details of axes notation), often driving the use of a SC unit-cell ${ }^{20-22}$ that aligns the four-screw single helices with the real-space lattice vectors, as shown in Figure 1b. It is the circular dichroism along these four-screw axes ${ }^{24}$ that enables applications such as chiral beam-splitting ${ }^{21}$, but with performance limited by a far from Bragg-like spectral response that includes sharp and narrow features, unpolarized reflection bands, as well as changes in the sign of circular dichroism ${ }^{20-22}$.

For optimal control of circular polarization, it is desirable for a nanostructure to exhibit Bragg-like circular dichroism, characterized by simple reflection of an LCP (RCP) incident wave with a spectral response equivalent to that of a Bragg mirror, and simultaneous complete transmission of a RCP (LCP) incident wave. In complex 3D nanostructures, smooth Bragg-like reflections often exist along the primitive reciprocal directions of the lattice. For the 1 srs nanostructure, the primitive directions that are parallel to the $\langle 110\rangle$ axes exhibit the broadest and cleanest Bragg-like band-gap (see Results and Discussion), a feature exploited by the butterfly Parides sesostris, which aligns the 1 srs $<110\rangle$ axes within specific patches of its wing-scales to maximize structural colouration ${ }^{35}$. The $\langle 110\rangle$ axes of the 1 srs nanostructure, however, do not exhibit sensitivity to circular polarization $^{25}$ and new structures must be investigated if Bragg-like circular dichroism is to be exploited.

The quadruple-gyroid 4 srs net ${ }^{24,32,36}$, which is comprised of four individual yet nonintersecting 1 srs nets of the same handedness, is a geometry that provides access to circularly polarized Bragg-like reflection bands by combining a SC primitive lattice type with cubic circular dichroism ${ }^{24}$. Building on the cubic symmetry of the 1 srs net, a 4srs net is formed via simple half-lattice translations of three duplicate 1srs nets - all of the same handedness-along each of the [100], [010] and [001] axes (Supplementary Fig. 1), ending in a 4srs net such as the inflated nanostructure shown in Figure 1c. In this process, the fourscrew single helices of the 1srs are transformed into pairs of fourscrew double helices ${ }^{24}$, and a new SC primitive unit cell is formed with cubic symmetry $P 4_{2} 32$, shown in Figure $1 \mathrm{~d}$. The length of the 4srs primitive unit cell is half the length of the non-primitive cubic 1 srs

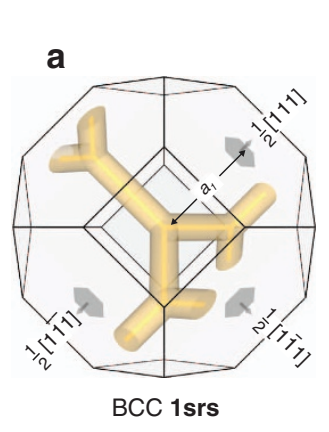

b

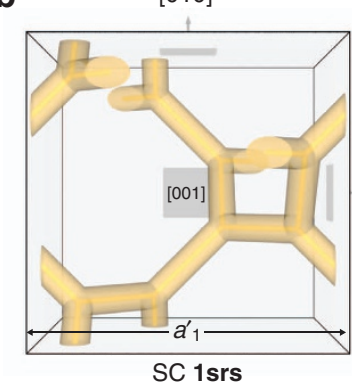

e

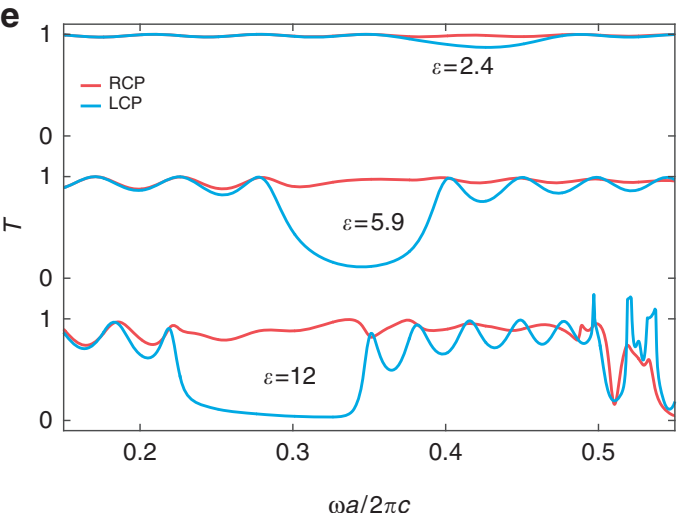

C
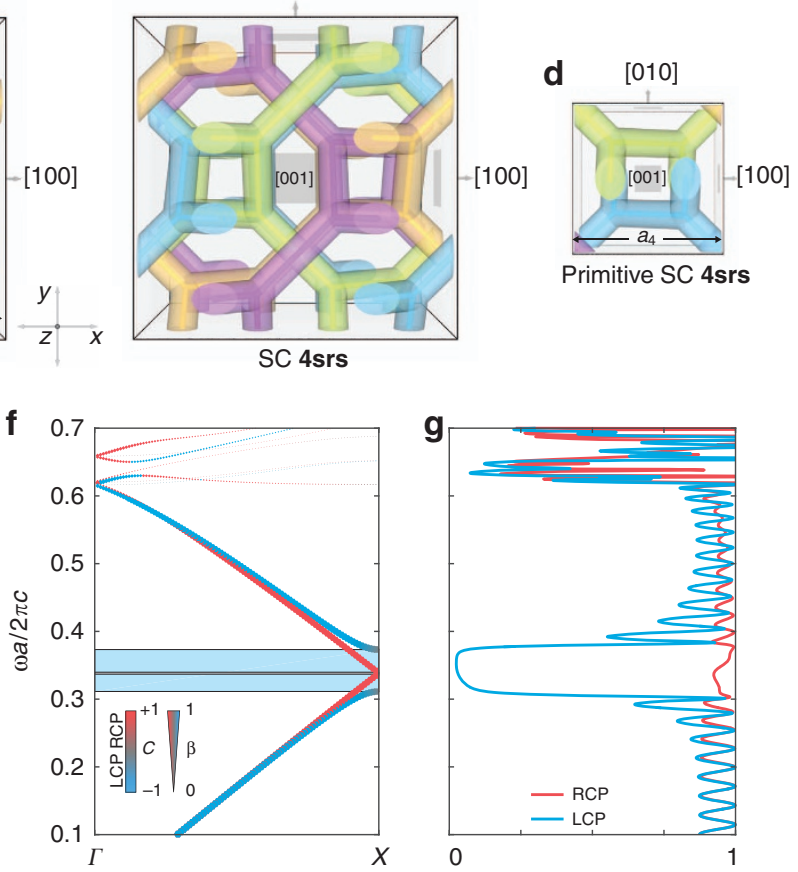

$|k|$

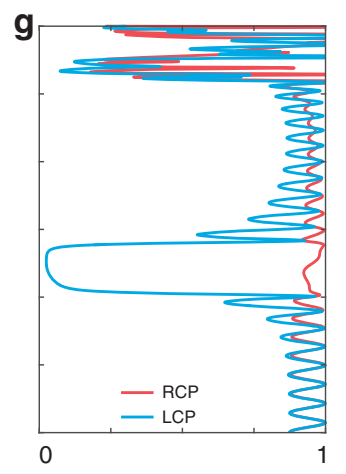

$T$

Figure 1 Geometry and numerical characterization of the quadruple-gyroid 4srs nanostructure. (a) A BCC unit cell of a RHD 1srs nanostructure (lattice parameter $a_{1}$ ). (b) A non-primitive cubic unit-cell of a RHD 1srs nanostructure (lattice parameter $a_{1}^{\prime}$ ) showing the four-screw helices along [001]. (c) A nonprimitive cubic unit-cell of a RHD 4srs nanostructure made of four identical 1srs nanostructures. (d) A primitive SC unit-cell of a RHD 4srs nanostructure (lattice parameter $a_{4}$ ) centred on the four-screw double helix. The grey patches and arrows indicate the direction of the real-space lattice vectors for each of the unit cells. (e) Simulated transmission spectra along the $<100>$ axes for 4 srs nanostructures with a height of six unit cells, a volume fraction of $\Phi=36 \%$ and permittivities of $\varepsilon=2.4, \varepsilon=5.9$ and $\varepsilon=12$. (f) Band-diagram $(\Gamma-X)$ and $(\mathrm{g})$ transmission spectra along the $<100>$ axes for a RHD 4 srs nanostructure with $\varepsilon=5.9, \Phi=36 \%$ and a height of 12 unit cells. The colour of the modes in $f$ represents the mode's circular dichroism index, $C$, while the linewidth of the plot indicates the mode's coupling index, $\beta$ (see Materials and Methods). The blue and grey shading indicates the location of the LCP stopband, and narrow band-gap, respectively. 
unit cell, even though the structural size of the four individual 1srs nets remain the same, a result of the translation operation pair-wise exchanging the four network domains of the 4srs.

\section{Numerical simulations}

The translation operations used to form the 4 srs nanostructure from a 1srs nanostructure induce two important transformations in its optical behaviour. First, the transformation of the primitive real-space lattice type from BCC to SC shifts the primitive reciprocal lattice vectors parallel to the $\langle 100\rangle$ axes (from parallel to the $\langle 110\rangle$ axes), ensuring that the Bragg-like reflection often associated with the primitive reciprocal lattice directions (see Results and Discussion) will be in a cubic basis. Second, the transformation of the four-screw single helices of the 1srs to the four-screw double helices of the 4srs enhances sensitivity to circular polarized light ${ }^{24}$ along the cubically symmetric $<100>$ axes. Figure 1e illustrates the Bragg-like circular dichroism properties that result from the combination of these transformations, with numerically simulated LCP and RCP transmission spectra (see Materials and Methods) along the [100] axis of a right handed (RHD) 4srs nanostructure plotted for the case of three relative 4srs permittivities of $\varepsilon=2.4, \varepsilon=5.9$ and $\varepsilon=12$ in a background of air $(\varepsilon=1)$, corresponding to the dielectric contrasts produced by common polymer ${ }^{16,20,37}$, chalcogenide glass $22,29,31$ and semiconductor ${ }^{38-40}$ fabrication techniques, respectively.

To characterize the circular dichroism of each transmission spectrum plotted in Figure 1e, we define the strength of circular dichroism as the differential transmission between LCP and RCP incident waves: $\Delta T=T_{\mathrm{RCP}}-T_{\mathrm{LCP}}$, and define the width of the circular dichroism band as the gap to mid-gap ratio: $\Delta \omega=2\left(\omega_{2}-\omega_{1}\right) /$ $\left(\omega_{2}+\omega_{1}\right)$, where $\omega_{1}$ and $\omega_{2}$ are the upper and lower frequency bounds of the dichroism band, respectively. Low-permittivity materials produce only weak $(\Delta T=11 \%)$ circular dichroism, limiting their practicality in experimental realization of the 4srs nanostructure. Materials with larger permittivities such as typical semiconductors allow significantly strong and broad $(\Delta T=92.5 \%, \Delta \omega=40 \%)$ circular dichroism bands to be opened, but at the cost of significant complexity in experimental realization ${ }^{38-40}$. Between these two extremes, chalcogenide glasses such as arsenic trisulfide $\left(\mathrm{As}_{2} \mathrm{~S}_{3}\right)$, which can be nanostructured in a similar manner to many low-permittivity polymers $22,29,31$, can also open broad bands of circular dichroism $(\Delta T=85.9 \%, \Delta \omega=22.9 \%)$ suitable for experimental realization.

As with previous simulations of topologically equivalent constant mean curvature 4 srs nanostructures ${ }^{24}$, the origin of the dichroism band in the cylindrical 4srs nanostructure is a splitting of the LCP and RCP mode frequencies surrounding the narrow band-gap along the $<100>$ axes $^{24}$. Figure if shows the numerically simulated bandstructure (see Materials and Methods) along the $<100>$ axes for a cylindrical RHD $\mathrm{As}_{2} \mathrm{~S}_{3}$ 4srs geometry with a dielectric volume fraction $(\Phi)$ of $36 \%$, and is plotted alongside a simulation of the transmission spectra in Figure $1 \mathrm{~g}$ for incident waves propagating through the same geometry. Each mode in the band-diagram in Figure $1 \mathrm{f}$ is analysed for its degree of circular dichroism and coupling amplitude (see Materials and Methods), plotted as the colour and linewidths of the curve, respectively. A clear separation of the first four LCP and RCP modes at the $\mathrm{X}$ point is evident, opening a LCP stop-band to create a band of circular dichroism 2.5 times wider than that produced by an equivalent RHD $\mathrm{As}_{2} \mathrm{~S}_{3}$ 1srs nanostructure (Supplementary Fig. 2). A full band-structure analysis of the 4 srs nanostructure can be found in Supplementary Fig. 3, highlighting the unique Bragg-like circular dichroism properties of the cubic $<100>$ axes.

\section{Laser nano-fabrication of 4srs nanostructures}

Unlike the 1srs geometry that is found ubiquitously in nature ${ }^{32}$ and which has been experimentally realized by means of selfassembly $^{37,41,42}$, bio-templating ${ }^{43,44}$, laser direct write techniques ${ }^{20-22}$ and $3 \mathrm{D}$ printing ${ }^{45}$, no 4 srs nanostructures have been either found or produced, although the occurrence of various triply intergrown geometries ${ }^{46-49}$ raises the hope that a self-assembly strategy for the 4srs will eventually be found. To experimentally realize the 4 srs nanostructure for the first time, we utilized the laser direct write technique in the chalcogenide glass $\mathrm{As}_{2} \mathrm{~S}_{3}$ to create cylindrical $\mathrm{As}_{2} \mathrm{~S}_{3}$ 4srs nanostructures in a three-step process as detailed in Figure 2a-2c a

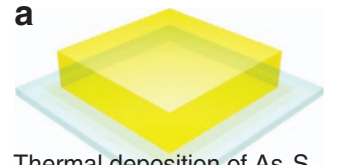

Thermal deposition of $\mathrm{As}_{2} \mathrm{~S}_{3}$

b

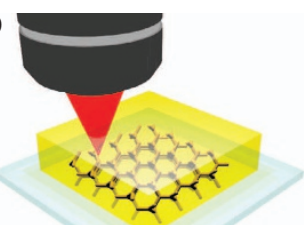

Local photo-polymerisation

C

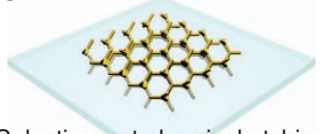

d
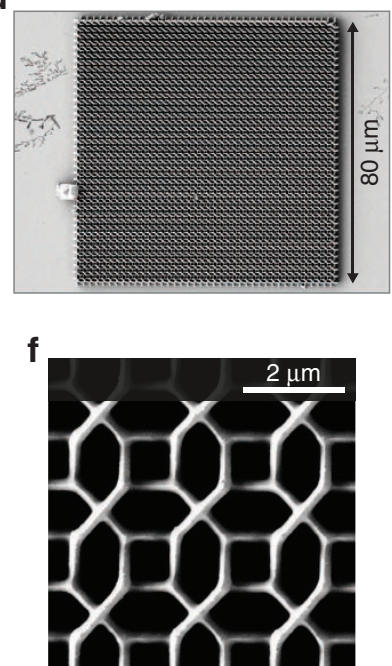

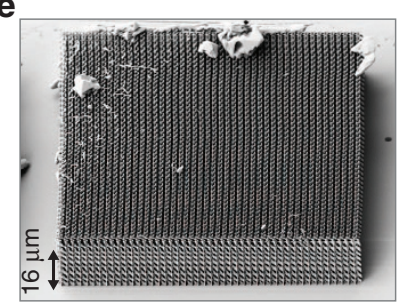

g



Figure 2 Three-step fabrication process of the $\mathrm{As}_{2} \mathrm{~S}_{3}$ laser direct write technique and fabricated $\mathrm{As}_{2} \mathrm{~S}_{3} 4 \mathrm{srs}$ nanostructures. (a) Thermal deposition of $A s_{2} \mathrm{~S}_{3}$ glass. (b) Local photo-polymerization with a focused femto-second laser beam, and (c) selective wet-chemical etching to reveal a free-standing nanostructure. (d) SEM image of a fabricated RHD 4srs nanostructure along the [001] axis. (e) SEM image of a fabricated RHD 4srs nanostructure along the [011] axis. (f, $\mathbf{g})$ are close-up images of the views in (d) and (e) showing the geometry of the four-screw double helices. 
and described in the Materials and Methods section. First, thick films of $\mathrm{As}_{2} \mathrm{~S}_{3}$ were deposited onto glass substrates using a controlled deposition procedure that preferentially deposits the $\mathrm{As}_{2} \mathrm{~S}_{3}$ as weakly interconnected $\mathrm{As}_{4} \mathrm{~S}_{6}$ cage molecules ${ }^{29,31}$. Second, the as-deposited films were locally and three-dimensionally photo-polymerized back to cross-linked $\mathrm{As}_{2} \mathrm{~S}_{3}$ with an adaptive optics enhanced direct laser writing system ${ }^{22}$. Third, the films were wet-chemically etched to enable preferential dissolution of the unexposed $\mathrm{As}_{4} \mathrm{~S}_{6}$, leaving a freestanding $\mathrm{As}_{2} \mathrm{~S}_{3}$ nano-structure with high dielectric contrast to the surrounding air.

Using this technique, 4srs nanostructures with primitive lattice parameters between $a_{4}=1.75$ and $2 \mu \mathrm{m}$ were fabricated with linewidths between 150 and $240 \mathrm{~nm}$ along the [100] and [010] axes, and between 0.9 and $1.2 \mu \mathrm{m}$ along the [001] axis, such that volume fractions covering the optimal value of $\Phi=19 \%$ (Supplementary Fig. 4h) were produced. In all cases, the nanostructures were oriented such that the [001] axis was perpendicular to the substrate surface and access to the four-screw double helices was possible.

Scanning electron microscope (SEM) images of fabricated 4srs nanostructures along the [001] and [011] axes are shown in Figure 2d and $2 \mathrm{e}$, with additional close-up images shown in Figure $2 \mathrm{f}$ and $2 \mathrm{~g}$ showing the characteristic four-screw double helices that separate the band-edge mode frequencies ${ }^{24}$ and lead to the opening of a circular dichroism band. The angled images along the [011] axis reveal a large degree of elongation of the network linewidth along the [001] axis. This elongation is the result of the elliptical shape of the diffraction limited focus in most laser writing systems, which for the system used here directly transforms the symmetric cylindrical segments of the 4srs to elliptic cylindrical segments with an ellipticity of $e=5$, where $e$ is defined by the ratio of the [001] to [100] lines widths of the elliptical line segments. Such elongation breaks the cubic symmetry of the 4srs nanostructure, and a variety of methods have been developed in order to restore $i^{21,22,31}$. However, as long as symmetry is maintained in the plane orthogonal to the [001] axis, the ellipticity will have negligible effect on the circular dichroism bands along the [001] axis (Supplementary Fig. 4) and hence preservation of cubic symmetry is not always necessary.

\section{Transmission spectroscopy of 4srs nanostructures}

To probe the circular dichroism of the 4srs nanostructures, Fourier transform infrared spectroscopy (see Materials and methods) was utilized to measure the circularly polarized transmission spectra across the mid-infrared wavelength range. The etched networks were mounted in the spectrometer such that the incident beam propagated parallel to the [001] axis, while the incident angle was limited to a narrow cone with a half apex angle $(\alpha)$ of $5^{\circ}$ as shown in the measurement geometry in Figure $3 \mathrm{a}$ and $3 \mathrm{~b}$. Figure $3 \mathrm{c}$ and $3 \mathrm{~d}$ shows the measured and simulated 4srs transmission spectra, respectively, for both LCP and RCP incident waves traversing a RHD 4srs nanostructure with $a_{4}=2 \mu \mathrm{m}$ and lateral and axial linewidths of 235 and $1,175 \mathrm{~nm}$, respectively, corresponding to a volume fraction of $\Phi=17.7 \%$. A strong reduction in transmission for the LCP incident beam is observed compared with the case of a RCP incident beam that propagates unaffected by the structure, resulting in the opening of a broad $(\Delta \omega=18 \%)$ and strong $(\Delta T=65 \%)$ band of Bragg-like circular dichroism. In comparison, the measured and simulated transmission a

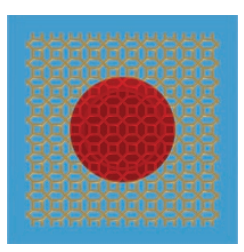

b

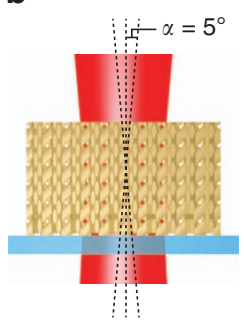

e

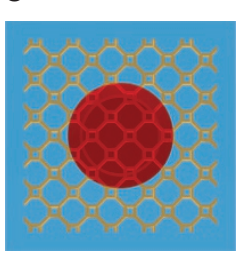

$\mathbf{f}$
C
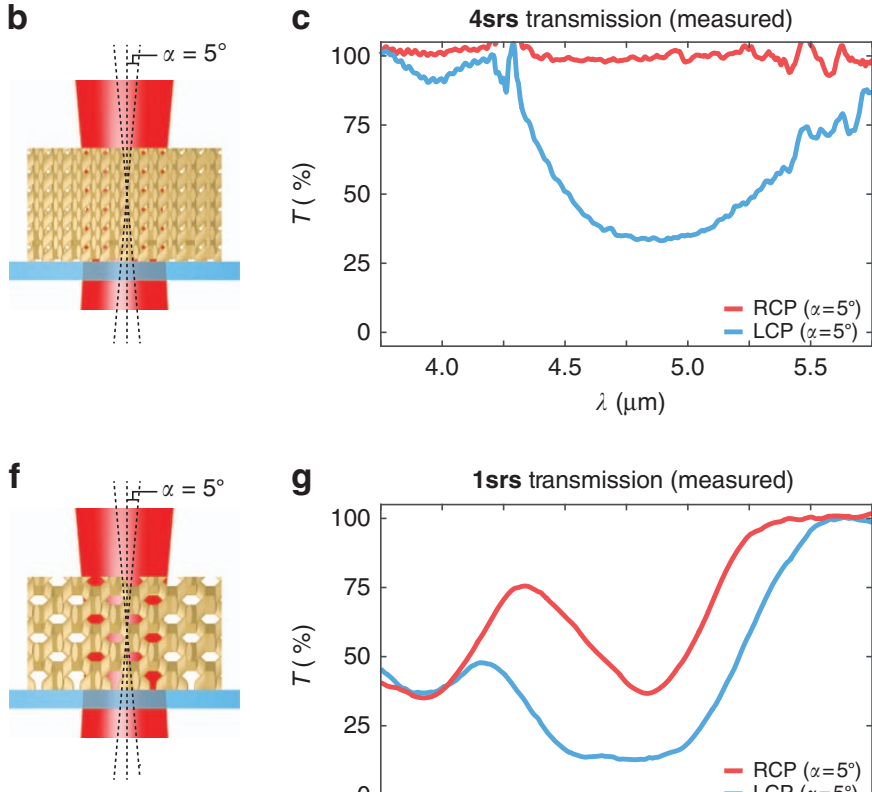

g



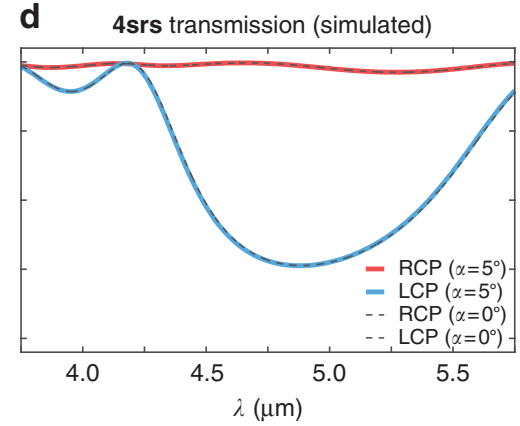

h

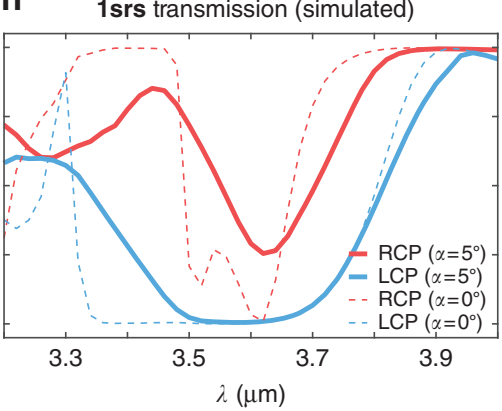

Figure 3 Experimental transmission characterization of the robust 4srs nanostructure circular dichroism and comparison with numerical simulations. (a, b) Transmission measurement geometry for the 4srs nanostructure. (c) Measured transmission spectra for a RHD 4srs nanostructure with a lattice parameter of $a_{4}=2 \mu \mathrm{m}$, a height of six unit cells, a lateral linewidth of $235 \mathrm{~nm}$ and $e=5$. (d) Simulated transmission spectra at normal incidence $\left(\alpha=0^{\circ}\right)($ dotted black lines) and at $\alpha=5^{\circ}$ (solid coloured lines) for a 4srs geometry equivalent to the experimental measurement. (e, f) Transmission measurement geometry for the 1srs nanostructure. (g) Measured transmission spectra for a RHD 1srs nanostructure with a lattice parameter $a_{1}^{\prime}=3 \mu \mathrm{m}$, a height of four unit cells, a lateral linewidth of $205 \mathrm{~nm}$ and $e=5$. (h) Simulated transmission spectra at normal incidence $\left(\alpha=0^{\circ}\right)$ (dashed thin lines) and at $\alpha=5^{\circ}$ (solid thick lines) for a 1 srs geometry equivalent to the experimental measurement. 
spectra for LCP and RCP incident waves traversing the [001] axis (Figure $3 \mathrm{e}$ and $3 \mathrm{f}$ ) of a 1srs nanostructure (see Supplementary Fig. 5 for SEM images) can be seen in Figure $3 \mathrm{~g}$ and $3 \mathrm{~h}$, respectively, where two considerably narrower bands of circular dichroism with a maximum width of $\Delta \omega=5.6 \%$ exist in a significantly more complex transmission spectra that includes dips in transmission for both leftand right-handed circularly polarized waves.

In addition to the presence of strong and broadband circular dichroism, comparison between the measured and simulated transmission spectra reveals a further advantage of the 4 srs nanostructure over similar $1 \mathrm{srs}$ nanostructures. For the case of the $4 \mathrm{srs}$ nanostructure, good agreement is found between the measured and transmission spectra when accounting for both the full $\alpha=5^{\circ}$ half apex angle of the incident beam, as well as when the simulation is limited to normal incidence $\left(\alpha=0^{\circ}\right)$. The 1 srs nanostructure however, shows significant deviation between the numerical simulations at normal incidence and at $\alpha=5^{\circ}$. This indicates excellent robustness of the 4 srs nanostructure to changes in both the incident angle, and to deviations from plane wave illumination surrounding the $[001]$ axis.

The origin of the Bragg-like characteristics of the circular dichroism band lies in the coincidence of the four-screw double-helix axes with the direction of the primitive reciprocal lattice vectors. Along these primitive directions, the first Brillouin zone is met with the shortest possible wave-vectors such that other reciprocal lattice vectors that possess much larger wave-vector lengths cannot contribute to the band structure. In such a situation, the scattering problem reduces approximately to a single-mode problem equivalent to the situation of a Bragg mirror or simple multilayer $\operatorname{stack}^{25}$ and accordingly, a simple band-gap with strong reflection is surrounded by a single pair of modes permitting high transmission is opened. In combination with the distinct topological continuities of opposite handed screw motions along the four-screw double helices ${ }^{24,25}$ that drive a large separation of LCP and RCP mode frequencies, the band-gap is transformed into a broad band of circular dichroism with a spectral response reminiscent of the clean broadband reflection from a Bragg mirror.

The origin of the robustness to incident angle arises from the SC symmetry of the 4srs, which translates to a SC Brillouin zone in reciprocal space, shown in Figure $4 \mathrm{a}$ and $4 \mathrm{~b}$. Real-space propagation along the $\langle 100\rangle$ axes corresponds to wave-vectors, $\mathbf{k}$, terminating at the face of the Brillouin zone, such that small changes to the incident angle, $\theta$, increase $|k|$ in quadratic order. Accordingly, the band-edge mode frequencies undergo only a minor blue-shift as shown in Figure 4c, which plots the spectral position of the circular dichroism band for the experimental 4srs geometry as a function of $\theta$. In contrast, the 1 srs has BCC symmetry regardless of the choice of experimentally convenient non-primitive cubic unit cell, resulting in a face centred cubic (FCC) Brillouin zone in reciprocal space, shown in Figure $4 \mathrm{~d}$ and 4 e. In this case, real-space propagation along the $\langle 100\rangle$ axes corresponds to wave-vectors that terminate at the Brillouin zone four-edge vertices, such that a small change in the incident angle result in a large decrease in $|k|$. Consequently, the band-edge mode frequencies undergo a strong red-shift as shown in Figure $4 \mathrm{f}$, which plots the spectral position of the experimental 1srs circular dichroism bands (see Supplementary Fig. 6 for a band-diagram of this geometry) as a function of $\theta$. For small angles of incidence, the disparate shift in mode frequencies between the two networks is present for all volume fractions and network ellipticities, as shown in Supplementary Fig. 7. This indicates that the switch from BCC to SC symmetry can provide robustness to angle of incidence in the presence of the most common geometric variations, so long as the topology of the network is preserved.

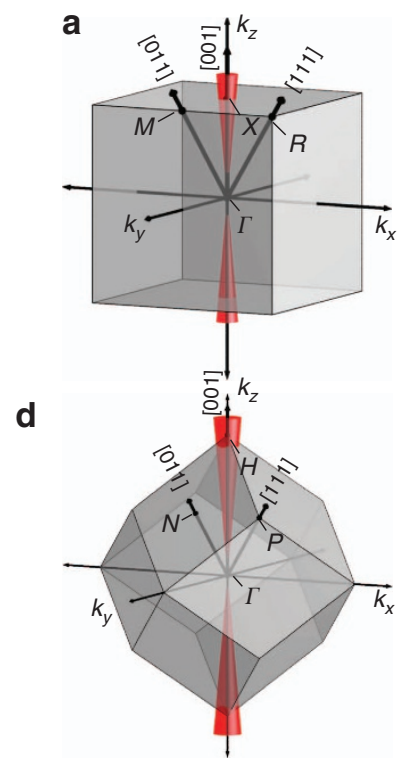

b
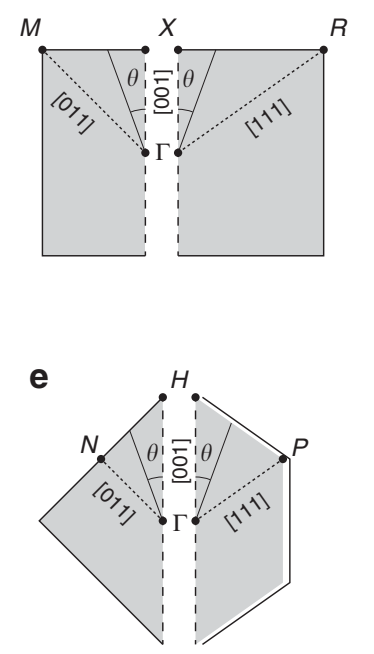

C
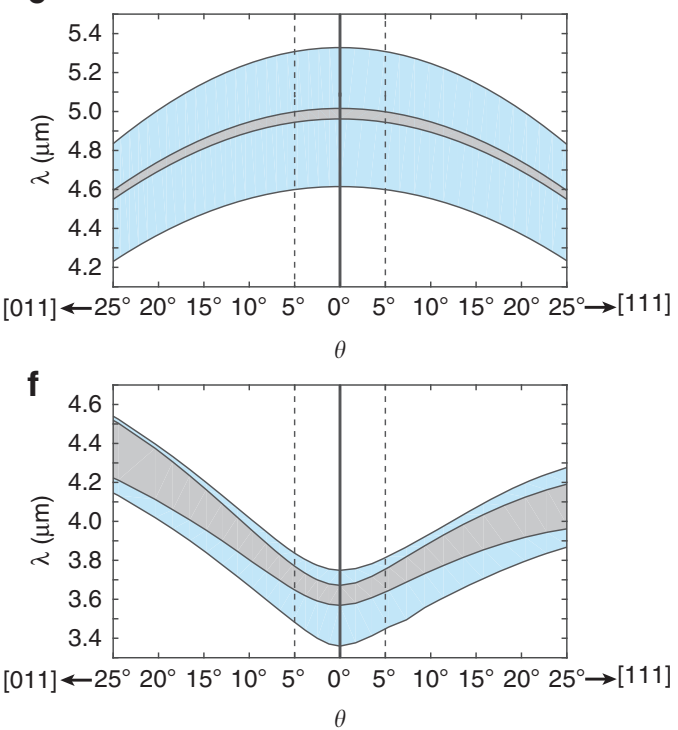

Figure 4 Origin of the robust circular dichroism in quadruple-gyroid 4srs nanostructures. (a) First Brillouin zone of a 4 srs nanostructure and the measured wave-vectors (red cone) within the $\alpha=5^{\circ}$ half apex angle around [001]. (b) Partial cross-sections of the $\Gamma-X-M$ and $\Gamma-X-R$ Brillouin zone planes for the 4srs nanostructure. (c) Plot of the 4srs nanostructure circular dichroism bands (blue shading) and band-gap (grey shading) spectral positions as a function of incident angle $(\theta)$ from [001] to [011] and [111]. (d) First Brillouin zone of the 1srs nanostructure and the measured wave-vectors (red cone) within the $\alpha=5^{\circ}$ half apex angle around [001]. (e) Partial cross-sections of the $\Gamma-H-N$ and $\Gamma-H-P$ Brillouin zone planes for the 1srs nanostructure. (f) Plot of the 1srs nanostructure circular dichroism band spectral position as a function of incident angle $(\theta)$ from [001] to both [011] and [111]. 
One possible application of the 4srs nanostructure is in the engineering of defect states ${ }^{50,51}$ within the circular dichroism band. In particular, quarter-twist planar defect layers ${ }^{52,53}$ can introduce a defect mode precisely at the mid-gap wavelength of a circular dichroism band $\mathrm{d}^{52,53}$. Supplementary Fig. 8 shows transmission spectra and SEM images of a fabricated RHD 4srs nanostructure with a quarter-twist defect layer in the (001) plane, creating a single defect mode within the circular dichroism band and producing a peak in LCP transmission that is pinned to the mid-gap wavelength. Such defects may be useful for tailoring the position and strength of circular dichroism bands ${ }^{52}$, or increasing the photonic density of states for chiral lasing ${ }^{51}$.

\section{CONCLUSION}

We have shown that the quadruple-gyroid 4 srs nanostructure is a remarkable nanostructure that provides an exceptional ability to filter broad bands of circularly polarized light with strong resilience against deviations in the angle of incidence and beam collimation, even when the nanostructure possesses asymmetric cylindrical segments. Furthermore, we have shown that the 4srs nanostructure allows for the creation of Bragg-like circular dichroism without spectral inconsistencies, unlike many other chiral structures that exhibit non-uniform circular dichroism and transmission ${ }^{13-22}$.

\section{CONFLICT OF INTEREST}

The authors declare no conflict of interest.

\section{ACKNOWLEDGEMENTS}

This research was conducted by the Australian Research Council Centre of Excellence for Ultrahigh bandwidth Devices for Optical Systems (project number CE110001018). We would like to thank Matthias Saba, Johannes Hielscher and Mark D. Turner for their useful discussions on the numerical simulations and experimental fabrication.

1 Rayleigh L. On the maintenance of vibrations by forces of double frequency, and on the propagation of waves through a medium endowed with a periodic structure. Phil Mag Ser 5 1887; 24: 145-159.

2 Joannopoulos JD, Johnson SG, Winn JN, Meade RD. Photonic Crystals: Molding the Flow of Light, 2nd edn Princeton University Press: Princeton. 2011.

3 Gong QH, Hu XY. Photonic Crystals: Principles and Applications. Pan Stanford Publishing: Stanford. 2014.

4 MacLeod HA, Macleod HA. Thin-Film Optical Filters, 4th edn. CRC Press: New York. 2010

5 Kashyap R. Fiber Bragg Gratings. Academic Press: New York. 2010

6 Carroll JE, Whiteaway J, Plumb D. Distributed Feedback Semiconductor Lasers. Institution of Electrical Engineers: London. 1998.

7 Noda S, Yokoyama M, Imada M, Chutinan A, Mochizuki M. Polarization mode control of two-dimensional photonic crystal laser by unit cell structure design. Science 2001; 293: 1123-1125.

8 O'Brien JL, Furusawa A, Vučković J. Photonic quantum technologies. Nat Photon. 2009; 3: 687-695.

9 Bozinovic N, Yue Y, Ren YX, Tur M, Kristensen P et al. Terabit-scale orbital angular momentum mode division multiplexing in fibers. Science 2013; 340: $1545-1548$.

10 D'Ambrosio V, Nagali E, Walborn SP, Aolita L, Slussarenko S et al. Complete experimental toolbox for alignment-free quantum communication. Nat Commun 2012; 3: 961.

11 Gansel JK, Thiel M, Rill MS, Decker M, Bade K et al. Gold helix photonic metamaterial as broadband circular polarizer. Science 2009; 325: 1513-1515.

12 Yang ZY, Zhao M, Lu PX. Improving the signal-to-noise ratio for circular polarizers consisting of helical metamaterials. Opt Express 2011; 19: 4255-4260.

13 Radke A, Gissibl T, Klotzbücher T, Braun PV, Giessen H. Three-dimensional bichiral plasmonic crystals fabricated by direct laser writing and electroless silver plating. Adv Mater 2011; 23: 3018-3021.

14 Kaschke J, Wegener M. Gold triple-helix mid-infrared metamaterial by STED-inspired laser lithography. Opt Lett 2015; 40: 3986-3989.
15 Thiel M, Hermatschweiler M, Wegener M, von Freymann G. Thin-film polarizer based on a one-dimensional-three-dimensional-one-dimensional photonic crystal heterostructure. App/ Phys Lett 2007; 91: 123515.

16 Thiel M, Decker M, Deubel M, Wegener M, Linden S et al. Polarization stop bands in chiral polymeric three-dimensional photonic crystals. Adv Mater 2007; 19: 207-210.

17 Thiel M, Rill MS, von Freymann G, Wegener M. Three-dimensional bi-chiral photonic crystals. Adv Mater 2009; 21: 4680-4682.

18 Thiel M, Fischer H, von Freymann G, Wegener M. Three-dimensional chiral photonic superlattices. Opt Lett 2010; 35: 166-168.

19 Kao T-H, Chien L-YC, Hung Y-C. Dual circular polarization gaps in helix photonic metamaterials. Opt Express 2015; 23: 24416-24425.

20 Turner MD, Schröder-Turk GE, Gu M. Fabrication and characterization of threedimensional biomimetic chiral composites. Opt Express 2011; 19: 10001-10008.

21 Turner MD, Saba M, Zhang QM, Cumming BP, Schröder-Turk GE et al. Miniature chiral beamsplitter based on gyroid photonic crystals. Nat Photon. 2013; 7: 801-805.

22 Cumming BP, Turner MD, Schröder-Turk GE, Debbarma S, Luther-Davies B et al. Adaptive optics enhanced direct laser writing of high refractive index gyroid photonic crystals in chalcogenide glass. Opt Express 2014; 22: 689-698.

23 Dolan JA, Wilts BD, Vignolini S, Baumberg JJ, Steiner U et al. Optical properties of gyroid structured materials: from photonic crystals to metamaterials. Adv Opt Mater 2015; 3: 12-32.

24 Saba M, Thiel M, Turner MD, Hyde ST, Gu M et al. Circular dichroism in biological photonic crystals and cubic chiral nets. Phys Rev Lett 2011; 106: 103902.

25 Saba M, Wilts BD, Hielscher J, Schröder-Turk GE. Absence of circular polarisation in reflections of butterfly wing scales with chiral gyroid structure. Mater Today 2014; 1: 193-208.

26 Lee JCW, Chan CT. Polarization gaps in spiral photonic crystals. Opt Express 2005; 13 : 8083-8088.

27 Oh SS, Demetriadou A, Wuestner S, Hess 0 . On the origin of chirality in nanoplasmonic gyroid metamaterials. Adv Mater 2013; 25: 612-617.

28 Saba M, Schröder-Turk GE. Bloch modes and evanescent modes of photonic crystals: weak form solutions based on accurate interface triangulation. Crystals 2015; 5: 14-44.

29 Nicoletti E, Zhou GY, Jia BH, Ventura MJ, Bulla D et al. Observation of multiple higherorder stopgaps from three-dimensional chalcogenide glass photonic crystals. Opt Lett 2008; 33: 2311-2313.

30 Choi D-Y, Madden S, Bulla D, Wang RP, Rode A et al. Thermal annealing of arsenic trisulphide thin film and its influence on device performance. J App/ Phys 2010; 107: 053106.

31 Wong S, Deubel M, Pérez-Willard F, John S, Ozin GA et al. Direct laser writing of threedimensional photonic crystals with a complete photonic bandgap in chalcogenide glasses. Adv Mater 2006; 18: 265-269.

32 Hyde ST, O'Keeffe M, Proserpio DM. A short history of an elusive yet ubiquitous structure in chemistry, materials, and mathematics. Angew Chem Int Ed Engl 2008; 47: 7996-8000.

33 Lu L, Fu L, Joannopoulos JD, Soljačić M. Weyl points and line nodes in gyroid photonic crystals. Nat Photon. 2013; 7: 294-299.

34 Lu L, Wang ZY, Ye DX, Ran LX, Fu L et al. Experimental observation of Weyl points. Science 2015; 349: 622-624.

35 Yoshioka S, Fujita H, Kinoshita S, Matsuhana B. Alignment of crystal orientations of the multi-domain photonic crystals in Parides sesostris wing scales. $J R$ Soc Interface 2013; 11: 20131029.

36 Wells AF. Three Dimensional Nets and Polyhedra. Wiley: New York. 1977.

37 Urbas AM, Maldovan M, DeRege P, Thomas EL. Bicontinuous cubic block copolymer photonic crystals. Adv Mater 2002; 14: 1850-1853.

38 Hermatschweiler M, Ledermann A, Ozin GA, Wegener M, von Freymann G. Fabrication of silicon inverse woodpile photonic crystals. Adv Funct Mater 2007; 17: 2273-2277.

39 Tétreault N, von Freymann G, Deubel M, Hermatschweiler M, Pérez-Willard F et al. New route to three-dimensional photonic bandgap materials: Silicon double inversion of polymer templates. Adv Mater 2006; 18: 457-460.

40 García-Santamaría F, Xu M, Lousse V, Fan S, Braun PV et al. A germanium inverse woodpile structure with a large photonic band gap. Adv Mater 2007; 19: 1567-1570.

41 Chan VZ-H Hoffman J, Lee VY, latrou H, Avgeropoulos A et al. Ordered bicontinuous nanoporous and nanorelief ceramic films from self assembling polymer precursors. Science 1999; 286: 1716-1719.

42 Hajduk DA, Harper PE, Gruner SM, Honeker CC, Kim G et al. The gyroid: a new equilibrium morphology in weakly segregated diblock copolymers. Macromolecules 1994; 27: 4063-4075.

43 Mille C, Tyrode EC, Corkery RW. 3D titania photonic crystals replicated from gyroid structures in butterfly wing scales: approaching full band gaps at visible wavelengths. RSC Adv 2013; 3: 3109-3117.

44 Mille C, Tyrode EC, Corkery RW. Inorganic chiral 3-D photonic crystals with bicontinuous gyroid structure replicated from butterfly wing scales. Chem Commun 2011; 47: 9873-9875.

45 Pouya C, Vukusic P. Electromagnetic characterization of millimetre-scale replicas of the gyroid photonic crystal found in the butterfly Parides sesostris. Interface Focus 2012; 2: 645-650.

46 Fischer MG, de Campo L, Kirkensgaard JJK, Hyde ST, Schröder-Turk GE. The tricontinuous 3 ths(5) phase: a new morphology in copolymer melts. Macromolecules 2014; 47: 7424-7430.

47 Han Y, Zhang DL, Chng LL, Sun JL, Zhao L et al. A tri-continuous mesoporous material with a silica pore wall following a hexagonal minimal surface. Nat Chem 2009; 1 : $123-127$. 
48 Sorenson GP, Schmitt AK, Mahanthappa MK. Discovery of a tetracontinuous, aqueous lyotropic network phase with unusual 3D-hexagonal symmetry. Soft Matter 2014; 10 8229-8235.

49 Schroder-Turk GE, de Campo L, Evans ME, Saba M, Kapfer SC et al. Polycontinuous geometries for inverse lipid phases with more than two aqueous network domains. Faraday Discuss 2013; 161: 215-247.

50 Braun PV, Rinne SA, García-Santamaría F. Introducing defects in 3D photonic crystals: state of the art. Adv Mater 2006; 18: 2665-2678.

51 Kopp VI, Zhang ZQ, Genack AZ. Lasing in chiral photonic structures. Prog Quant Electron 2003; 27: 369-416.

52 Hodgkinson IJ, Wu QH, Thorn KE, Lakhtakia A, McCall MW. Spacerless circularpolarization spectral-hole filters using chiral sculptured thin films: theory and experiment. Opt Commun 2000; 184: 57-66.
53 Kopp VI, Genack AZ. Twist defect in chiral photonic structures. Phys Rev Lett 2002; 89: 033901.

(c) (i) $(-)$ This work is licensed under a Creative Commons Attributioncc) NonCommercial-NoDerivs 4.0 International License. The images or other third party material in this article are included in the article's Creative Commons license, unless indicated otherwise in the credit line; if the material is not included under the Creative Commons license, users will need to obtain permission from the license holder to reproduce the material. To view a copy of this license, visit http:// creativecommons.org/licenses/by-nc-nd/4.0/

(C) The Author(s) 2017

Supplementary Information for this article can be found on the Light: Science \& Applications' website (http://www.nature.com/lsa). 